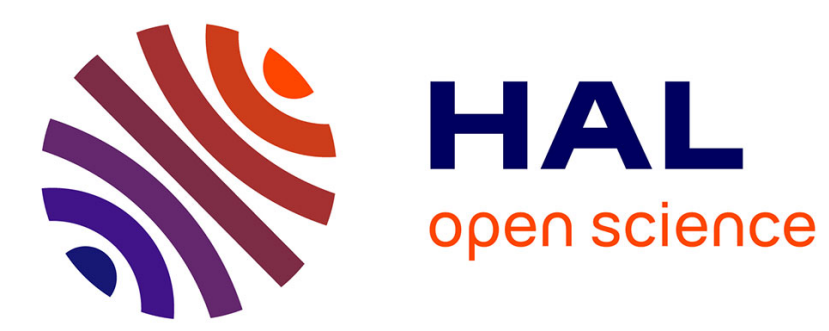

\title{
MICRO-ANALYSE DE CRISTAUX EN CROISSANCE SUR UNE SURFACE DE SILICIUM
}

J. Beauvillain, Alain Claverie, Yolande Kihn, Jean Sévely, B. Jouffrey

\section{To cite this version:}

J. Beauvillain, Alain Claverie, Yolande Kihn, Jean Sévely, B. Jouffrey. MICRO-ANALYSE DE CRISTAUX EN CROISSANCE SUR UNE SURFACE DE SILICIUM. Journal de Physique Colloques, 1984, 45 (C2), pp.C2-657-C2-660. 10.1051/jphyscol:19842153 . jpa-00223825

\section{HAL Id: jpa-00223825 https://hal.science/jpa-00223825}

Submitted on 1 Jan 1984

HAL is a multi-disciplinary open access archive for the deposit and dissemination of scientific research documents, whether they are published or not. The documents may come from teaching and research institutions in France or abroad, or from public or private research centers.
L'archive ouverte pluridisciplinaire HAL, est destinée au dépôt et à la diffusion de documents scientifiques de niveau recherche, publiés ou non, émanant des établissements d'enseignement et de recherche français ou étrangers, des laboratoires publics ou privés. 


\title{
MICRO-ANALYSE DE CRISTAUX EN CROISSANCE SUR UNE SURFACE DE SILICIUM
}

\author{
J. Beauvillain, A. Claverie, Y. Kihn, J. Sevely et B. Jouffrey \\ Iaboratoire d'optique Electronique du C.N.R.S., 29 me Jeanne Marvig, \\ 31055 Touzouse Cedex, France
}

\begin{abstract}
Résumé : Sur une surface de silicium, préalablement polie, chauffée dans un microscope êlectronique, nous avons fait crỗtre des cristaux que nous avons observēs et analysēs.
\end{abstract}

Abstract : Crystals were grown on a polished silicon surface by heating in an electron microscope column. These crystals and their growths were studied by TEM and analysed by EELS, revealing structure and composition.

Ces dernières années, la technique d'observation des surfaces en micrsocopie électronique par réflexion a connu un nouveau développement (1), (2), (3). Nous avons repris ces travaux et essayé d'observer des états de surfaces propres dans un vide relativement modeste $\left(10^{-5}, 10^{-6}\right.$ Torr $)$. Avec du Silicium, la technique couramment utilisée pour obtenir une surface propre est une "reconstruction" de la surface par chauffage (4), (5).

Au cours de telles opérations, nous avons observé la formation de cristaux dont la forme, la taille et la structure dépendent de la température à laquelle ils sont crêés. Nous pouvons différencier trois types de cristallisations différentes

$\left(t>800^{\circ} \mathrm{C}\right)$ :
- La première se fait sous forme de pyramides (base $\sim q q \mu^{2}$ ), très transparentes aux électrons, mais très instables (Fig. 1).

- Un chauffage prolongé, à une température légèrement supérieure, fait apparaître des cristaux sous forme de grosses fibres $(\emptyset \sim 5000 \AA)$ qui croissent perpendiculairement à la surface (Fig. 2).

- A une température plus élevée encore $\left(\sim 900^{\circ} \mathrm{C}\right)$, la surface est recouverte de nombreuses fibres dont le diamètre est inférieur à $1000 \AA$ (Fig. 3). Ces fibres peuvent, suivant la tempërature, se recombiner entre elles pour constituer, soit des fibles plus épaisses (Fig. 4), soit des cristaux contenant de nombreuses mâcles.

A plus haute température $\left(>1000^{\circ} \mathrm{C}\right)$, $i 1$ apparaît des excroissances linéaires courbées et terminées à leur extrémité par une boule, signalant un mécanisme de formation de type V.L.S. (6) (Fig. 5).

Nous avons analysé ces fibres par spectromëtrie de pertes d'énergie d'électrons (7), (8), (9) au cours de leur formation dans le microscope électronique de 1 MeV de Toulouse. Les spectres de pertes d'énergie montrent que toutes les structures cristallisées sont composées d'oxygēne et de Silicium qui peuvent caractēriser le dioxyde de Silicium. L'étude des diagrammes de diffraction électronique fait apparầtre différentes structures dont une seule a été identifiée (Fig. 3 et 4 ). La microanalyse nous a permis aussi de connaître la composition des excroissances obtenues à haute température par un mécanisme de type V.L.S., il s'agit d'un mélange de Nickel, de Fer et d'oxygène ; l'extrémité étant exclusivement constituée de Nickel pur provenant de la grille support de l'objet.

\section{Réfërences}

(1) C. FERT, Bul1. de Microsc. App 1. $6 n^{\circ} 3,1956,68-72$.

(2) N. OSAKABE, Y. TANISHIRO, K. YAGI, G. HONJO, Surface Science 102, 1981, 424-42.

(3) Z.C. KANG, J. Microsc. Spectrosc. Electron. 7, 1982, 33-39.

(4) S. INO, Jap. J. Applied Physics $16 n^{\circ} 6,197 \overline{7}, 891-908$. 
(5) N. OSAKABE, Y. TANISHIRO, K. YAGI, G. HONJ0, Surface Science 97, 1980, 393-408

(6) R.S. WAGNER, W.C. ELLIS, K.A. JACKSON, S.M. ARNOLD, J. Applied Phys. $35 n^{\circ} 10$, $1964,2993-3000$.

(7) J. SEVELY, Le vide $191,1978,69-75$.

(8) B. JOUFFREY, Y. KIHN, J.Ph. PEREZ, J. SEVELY et G. ZANCHI, 9th Int. Cong. Elec. Mic., III, Toronto 1978, 292-303.

(9) B. JOUFFREY et J. SEVELY, Rev. Phys. App1., 11, 1976, 101-111.

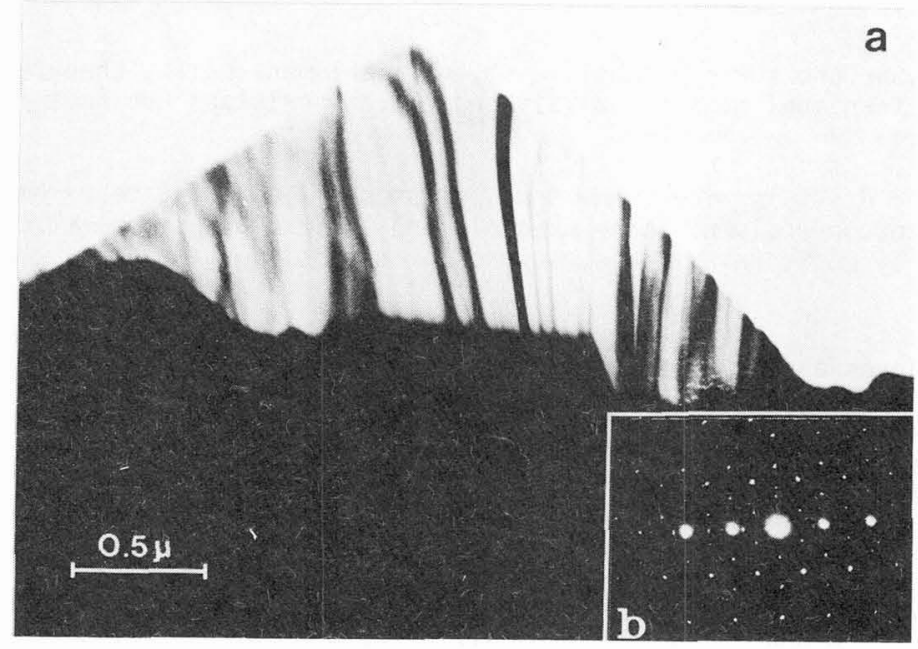

Fig. $1: T \cong 800^{\circ} \mathrm{C}$. Croissance d'un cristal sur une surface de silicium massif. La microanalyse quantitative par EELS a donné une composition de $\mathrm{Si}$ et $\mathrm{O}_{2}$ : a) image - b) diagramme de microdiffraction

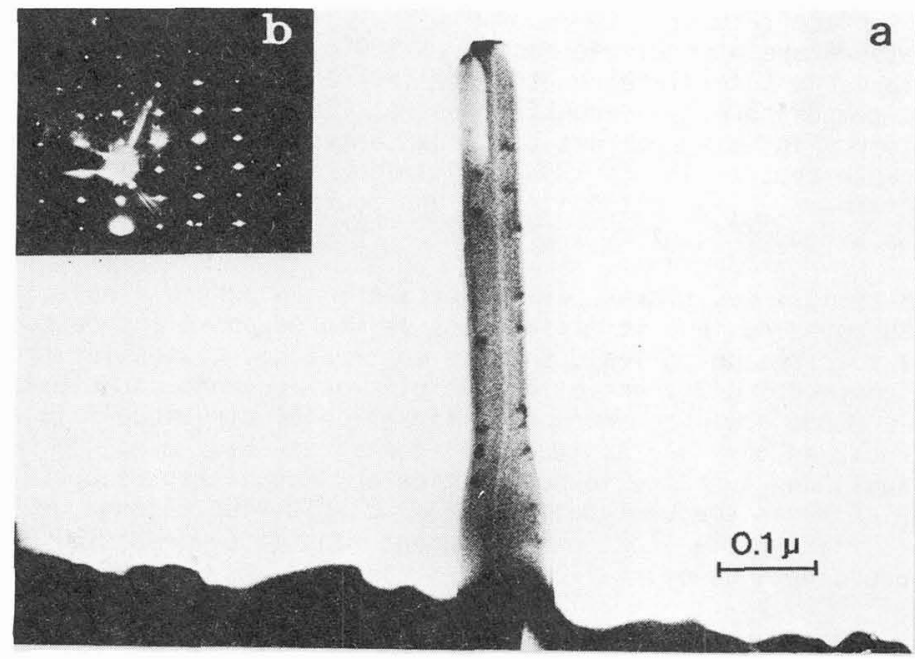

Fig. 2 : $800^{\circ} \mathrm{C}<\mathrm{T}<900^{\circ} \mathrm{C}$. Croissance sur une surface de silicium massif d'un cristal. Sa microanalyse qualitative par EELS a donné pour composition $\mathrm{Si}+0+$ quelques traces de $\mathrm{Fe}: \mathrm{a})$ image - b) diagramme de microdiffraction 


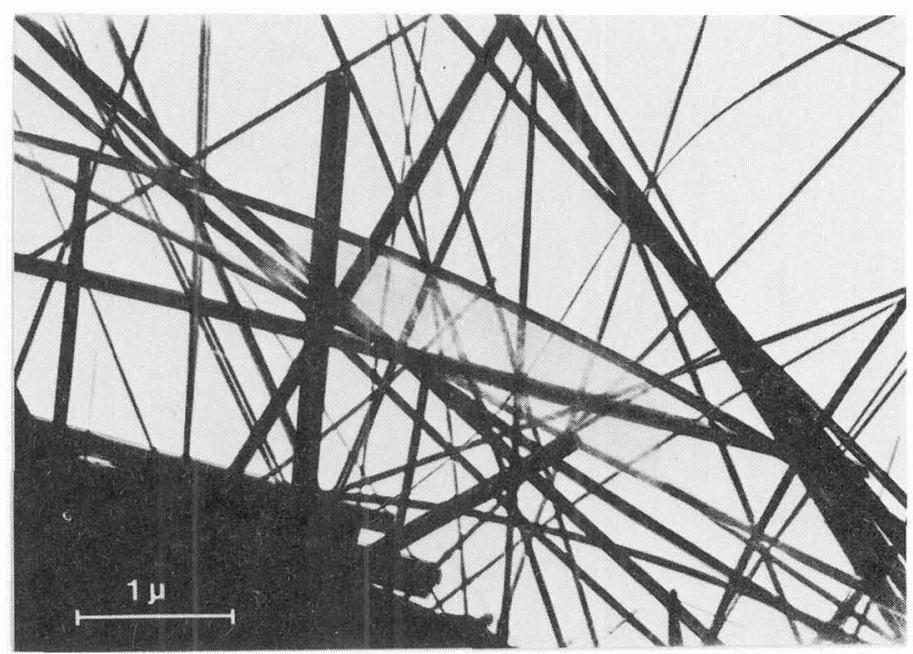

Fig. $3: T \simeq 900^{\circ} \mathrm{C}$. Croissance de silice fibreuse sur la surface de silicium massif. Ces cristaux ont été caractésirés par microanalyse et par indexation de microdiffraction

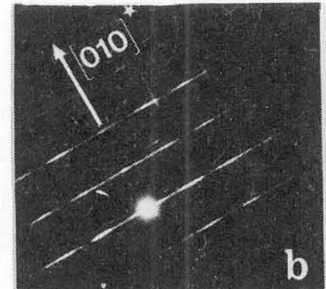

a

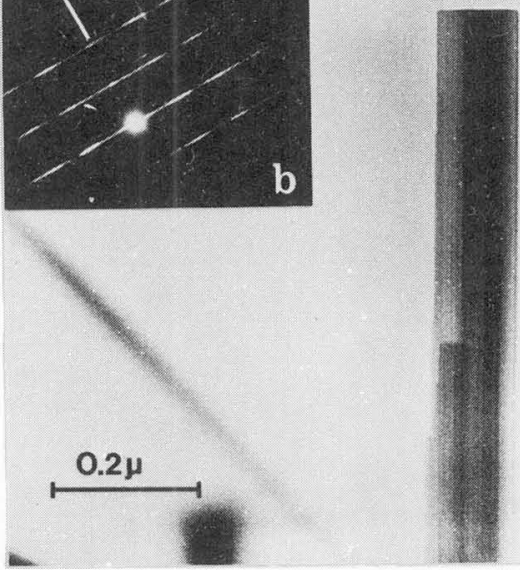

Fig. $4: T \simeq 900^{\circ} \mathrm{C}$. Fibres de silice - a) image - b) diagramme de diffraction - 


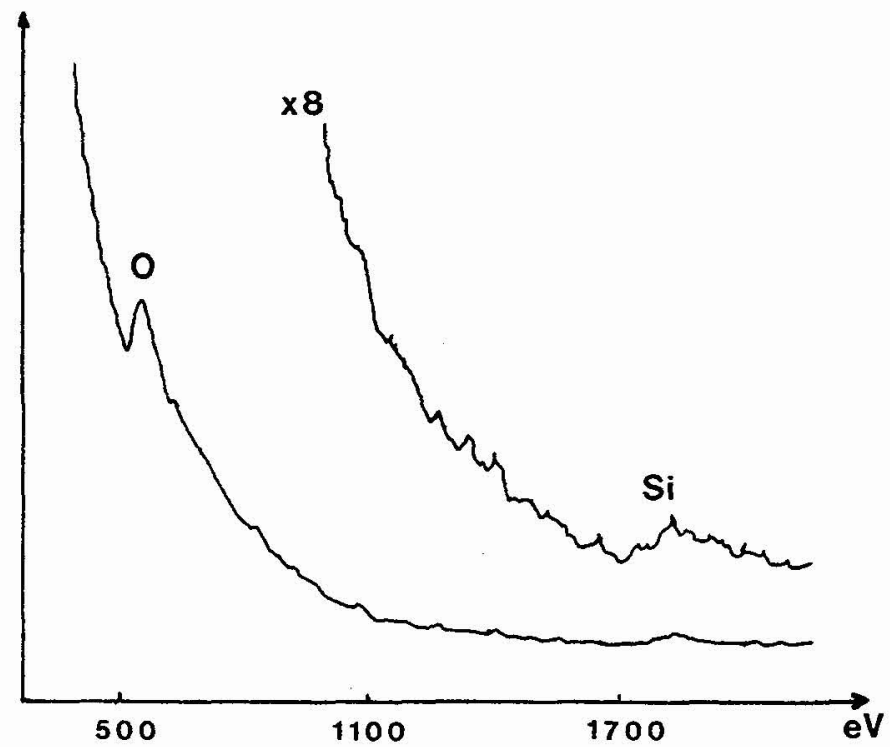

Fig. 3 et 4 bis : $T \simeq 900^{\circ} \mathrm{c}$. Fibre de Silice. Spectre de perte d'énergie d'électron (Unité arbitraire selon l'axe vertical).

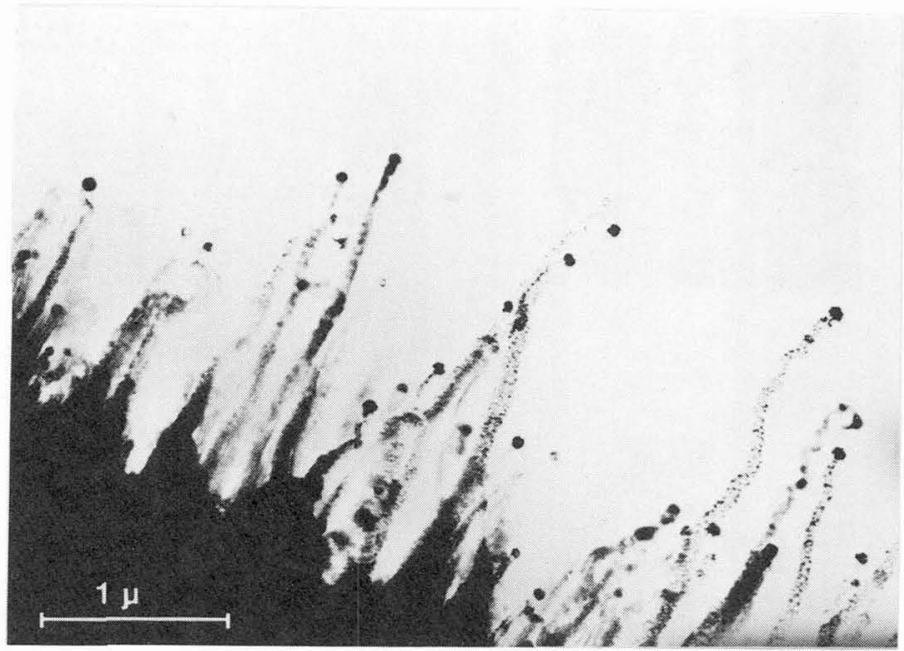

Fig. 5 : $T>1000^{\circ} \mathrm{C}$. Excroissances linéaires courbées et amorphes en formation sur une surface de silicium massif. Leur microanalyse par EELS a donné : - pour la fibre $\mathrm{O}+\mathrm{Fe}+\mathrm{Ni}$ - pour la boule noire du sommet : $\mathrm{Ni}$ 